\title{
Biométrie spatiale dans l'écosystème souterrain: répartition de Meta menardi Latr. (Argiopidae)
}

\author{
par
}

\section{R.R.TERCAFS ${ }^{1}$}

\section{INTRODUCTION}

L'intérêt des écologistes pour la distribution spatiale des organismes est assez ancienne puisque, dès les années 1920, des études ont été consacrées à ce sujet (par exemple, Arrhenius, 1921; Gleason, 1922). Les études récentes (Neyman et al., 1957; Piélou, 1961); Evans, 1969) ont porté, notamment, sur les types de répartition possible: aggrégation, répartition uniforme et aléatoire. Ces notions sont très importantes lorsque l'on veut établir des modèles mathématiques valables (Skellam, 1951).

Peu d'études ont été consacrées à ces problèmes de biométrie spatiale dans le cas du domaine souterrain. Or, ce milieu est extrêmement favorable à ce type de travaux puisque les populations cavernicoles ont des densités relativement faibles et vivent dans des milieux simplifiés (absence de végétaux). C'est pourquoi nous avons effectué une étude sur la distribution d'une Araignée troglophile répandue dans toute l'Europe, ainsi qu'en Amérique du Nord, Meta menardi Latr. (Argiopidae).

\section{MATÉRIEL ET MÉTHODES}

L'espèce cavernicole observée, Meta menardi, est très commune dans les grottes de Belgique. Elle construit des toiles lâches sur les parois des couloirs d'entrées. Elle capture ainsi des animaux rampant sur les parois, principalement des Myriapodes et des Crustacés terrestres (Tercafs, 1960). Les adultes se déplacent très peu et il est très facile de les observer.

L'intérêt principal des observations qui ont été effectuées est qu'elles englobent la totalité d'une population distribuée au sein d'un habitat homogène. En effet, les mesures portent sur des individus recensés sur une entité bien délimitée, aux caractéristiques physiques bien tranchées. Exemple: la première salle d'une caverne à entrée étroite. Dans ces conditions, et vu le petit nombre d'individus vivant dans cet habitat, il est matériellement possible d'enregistrer la position de tous les animaux présents.

Le recensement est effectué en mesurant la position des individus, en coordon-

\footnotetext{
${ }^{1}$ Chercheur qualifié du. Fonds National belge de la Recherche Scientifique. (Institut Royal des Sciences Naturelles 31 rue Vautier, Bruxelles, Belgique )
} 
nées cartésiennes, par rapport à un point arbitraire choisi à la frontière inférieure de l'habitat étudié.

Les zones répertoriées sont situées dans les cavernes suivantes (Belgique, province de Liège):

- Grotte de la Belle Roche, Comblain-au-Pont, couloir aux Araignées, 3/1/72.

- Grotte de Ramioul, Ramioul, galerie Louis, 22/1/72.

- Grotte Vampire, Comblain-au-Pont, grande salle, 26/1/72.

- Trou Mantô, Huy, petite galerie, 3/2/72.

- Trou Mantô, Huy, grande galerie, 3/2/72.

- Grotte des Racines, Huy, salle centrale, 3/2/72.

- Grotte St-Etienne, Huy, première salle, 3/2/72.

- Grotte de l'Amblève, Comblain-au-Pont, couloir d'entrée, 7/2/72.

- Grotte Steinlein, Comblain-au-Pont, partie gauche, première salle, 7/2/72.

- Grotte Steinlein, partie droite, couloir d'entrée, 7/2/72.

La position géographique de ces cavernes est détaillée dans les travaux de Tercafs (1961) et Vandersleyen (1967).

Les données brutes receuillies sont analysées par un programme écrit en Fortran et exécuté en ordinateur (IBM 360/44, couplé en multiprogrammation à un IBM 360/65, au Centre de Calcul de 1'Université de Liège, Belgique). Ce programme est constitué de deux parties:

\section{a) test de groupement}

- calcul d'une matrice des distances de chaque individu par rapport à chacun des autres.

- constitution de groupes, en calculant la somme des carrés des distances intérieures, en effectuant la moyenne de ces résultats, en conservant le groupe qui donne la moyenne la plus petite.

\section{b) calcul du $R$}

On utilise le test proposé par Clark et Evans (1954) qui consiste à utiliser la distance au plus proche voisin comme une mesure des relations spatiales au sein d'une population. On définit un coefficient $\mathrm{R}$ qui va être une estimation de la nature de la distribution des individus. Considérons ainsi une population constituée de $\mathrm{N}$ individus, peuplant une certaine surface à la densité $\mathrm{d}$, chacun séparé de son plus proche voisin par la distance $\mathrm{r}$. La distance moyenne au plus proche voisin est donc $r_{M}=\Sigma r / N$. Si la population est distribuée au hasard, $r_{H}=1 /(2 \sqrt{ } d)$. Ainsi, le rapport $\mathrm{R}=\mathrm{r}_{\mathrm{M}} / \mathrm{r}_{\mathrm{H}}$ peut être utilisé comme une mesure de la nature de la distribution spatiale. Si $R=1$, la population est distribuée au hasard; $R=2,146$ si chaque individu est équidistant de 6 de ses voisins; si tous les individus se rassemblent en un même point, $\mathrm{R}$ tend vers zéro.

a) test de groupement

\section{RÉSULTATS}

Chaque grotte a été analysée en détail. Il n'apparaît aucun groupement net au sein 
de chacune des populations. Le tableau 1 donne un exemple de résultat en considérant la grotte Steinlein, partie gauche.

Tableau 1.- Resumé des résultats d'un test de groupement en considérant la moyenne de la somme des carrés des distances entre animaux voisins. Le groupe qui a la moyenne la plus petite est conservé.

I et $\mathrm{J}$ désignent les $\mathrm{n}^{\mathrm{O}}$ des individus considérés à chaque calcul successif.

Value désigne la valeur de la fonction au stade considéré (Step).

\section{STEP GRAF OF GROUPING}

\section{ITEMS GROUPED}

$\begin{array}{rrr}\text { STEP } & \text { I } & \text { J } \\ 1 & 10 & 11 \\ 2 & 1 & 2 \\ 3 & 4 & 6 \\ 4 & 7 & 8 \\ 5 & 6 & 5 \\ 6 & 13 & 14 \\ 7 & 8 & 10 \\ 8 & 2 & 4 \\ 9 & 11 & 12 \\ 10 & 12 & 9 \\ 11 & 5 & 3 \\ 12 & 3 & 7 \\ 13 & 9 & 13\end{array}$

VALUE

$0.10100 \mathrm{E} \quad 03$

$0.22100 \mathrm{E} \quad 03$

$0.32500 \mathrm{E} \quad 03$

$0.45800 \mathrm{E} \quad 03$

$0.88333 \mathrm{E} \quad 03$

$0.16040 \mathrm{E} \quad 04$

$0.17965 \mathrm{E} \quad 04$

$0.23356 \mathrm{E} \quad 04$

$0.29078 \mathrm{E} \quad 04$

$0.39011 \mathrm{E} \quad 04$

$0.46683 \mathrm{E} \quad 04$

$0.77300 \mathrm{E} \quad 04$

$0.14195 \mathrm{E} \quad 04$

\section{$\begin{array}{llll}1 & 5 & 10 & 13\end{array}$ \\ $\begin{array}{llll}2 & 3 & 11 & 14\end{array}$}

$4 \quad 7 \quad 12$

$\begin{array}{cccc}1 & 5 & 10 & 13 \\ 2 & 3 & 11 & 14 \\ 4 & 7 & 12 & \\ 6 & 8 & 9\end{array}$

\section{b) Calcul de $R$}

Le tableau 2 donne les résultats obtenus dans chacune des cavités.

Tableau 2.- Estimation de la distribution de Meta menardi dans 10 biotopes souterrains. Le paramètre $\mathrm{R}$ désigne le rapport $(\Sigma \mathrm{r} / \mathrm{N}) /(1 / 2 \sqrt{ } \mathrm{d})$ où $\mathrm{r}=$ distance au plus proche voisin, $\mathrm{N}=$ nombre d'individus, $\mathrm{d}=$ densité de la population. $\mathrm{R}=$ valeur moyenne \pm écart-type. 


\begin{tabular}{|c|c|c|}
\hline Nom de la grotte & Nb. d'individus & $\mathrm{R}$ \\
\hline Trou Mantô, petite galerie & 7 & 1.21 \\
\hline Trou Mantô, grande galerie & 7 & 0.99 \\
\hline Grotte des Racines & 9 & 1.05 \\
\hline Grotte St-Etienne & 8 & 1.19 \\
\hline Grotte de l'Amblève & 12 & 0.62 \\
\hline Grotte Steinlein, gauche & 14 & 1.01 \\
\hline Grotte Steinlein, droite & 13 & 0.84 \\
\hline Grotte de Ramioul & 20 & 1.22 \\
\hline Grotte Vampire & 8 & 1.49 \\
\hline \multirow{2}{*}{$\begin{array}{c}\text { Grotte de la Belle Roche } \\
\text { total }\end{array}$} & 15 & 0.99 \\
\hline & 113 & $\mathrm{R}=1.061 \pm$ \\
\hline
\end{tabular}

Le calcul de la probabilité à l'équivalence des moyennes en utilisant le test classique de Student montre que le $\bar{R}$ n'est pas différent de $1(0.5>t>0.2)$.

\section{DISCUSSION ET CONCLUSION}

Des mesures de distribution ont été éffectuées sur 113 individus de l'espèce Meta menardi répartis en 10 populations différentes peuplant 8 cavités. Les tests de groupement ont donné des résultats négatifs: les animaux ne forment pas des amas. Ce résultat est confirmé lorsqu'on étudie les distances de chaque individu à son plus voisin (test du R de Clark et Evans, 1954). Les valeurs trouvées ne sont pas significativement différentes de 1 , ce qui indique que les animaux sont distribués au hasard. Cette constatation est très importante car la plupart des modèles mathématiques représentant l'évolution des populations animales ne sont valables que si une partie des organismes au moins est distribuée de façon aléatoire (Tercafs, 1971). Dans le cas étudié ici, les mesures ont pu être faites avec grande précision car l'espèce considérée n'est pas très abondante et, en plus, les individus restent immobiles pendant de longues périodes. Il est évident que cette répartition aléatoire n'existe qu'au sein du biotope où vivent les Araignées. A l'échelle de la cavité, les individus sont, bien entendu, groupés à l'endroit où les conditions climatiques et biologiques leur sont favorables.

Une étude analogue a été effectuée auparavant par Graham (1968) sur le Lépidoptère trogloxène Triphosa haesitata, mais avec des résultats très variables, dus vraisemblablement au fait que les zones étudiées n'étaient pas homogènes. 


\section{RÉSUMÉ}

La distribution spatiale de 113 individus de l'espèce Meta menardi Latr. (Argiopidae) répartis en 10 populations différentes peuplant 8 cavernes de Belgique a été étudiée. On utilise un test de groupement ainsi que le calcul du $\mathrm{R}$ de Clark et Evans (1954) basé sur la mesure de la distance au plus proche voisin. Les résultats montrent que les individus sont répartis au hasard au sein de leur biotope.

\section{SUMMARY}

The spatial distribution of 113 individuals from the species Meta menardi Latr. (Argiopidae) divided in 10 different populations living in 8 Belgian caves has been studied. A grouping test and the calculation of $\mathrm{R}$ according to Clark et Evans (1954), based on the measurement of the distance to the nearest neighbour, have been used. Results show that the individuals are distributed at random inside their biotope.

\section{BIBLIOGRAPHIE}

ARRHENIUS, O. 1921.- Species and area. J. Ecol., 9, 95-99.

CLARK, P.J. et EVA.NS, F.C. 1954.- Distance to the nearest neighbour as a measure of spatial relationships in populations. Ecology, 35, 445-453.

EVANS, C. 1966.- Space relations in ecology: an overview. Michigan Acad., 2, 69-76.

GLEASON, H.A. 1922. - On the relation between species and area. Ecology, 3, 158-162.

GRAHAM, R.E. 1958.- Spatial biometrics of subterranean demes of Triphosa haesitata (Lepidoptera: Geometridae). Caves and Karst, 10, 21-29.

NEYMAN, J. et SCOTT, E.L. 1957.- On a mathematical theory of populations conceived as a conglomeration of clusters. Cold Spring Harbor Symposia on quantitative Biology, 22, 109-120.

PIELOU, E.C. 1960.- A single mechanism to account for regular, random and aggregated populations. J. Ecology, 148, 575-584.

SKELLAM, J.G. 1951.-- Random dispersal in theoretical populations. Biometrika, 38, 196-218.

TERCAFS, R.R. 1960.- Notes à propos de deux araignées cavernicoles Meta menardi Latr. et Nesticus ceilulanus Clerk (Argiopidae). Ann. Féd. Spéléo. Belg., 1, 14-18.

TERCAFS, R.R. 1961.- Comparaison entre les individus épigés et cavernicoles d'un Mollusque gastéropode troglophile Oxychilus cellarius Müll. Ann. Soc. Roy. Zool. Belg., 91, 85-116.

TERCAFS, R.R. 1971.- Ecologie théorique. Simulation d'un système écologique de 6 populations animales vivant dans deux zones climatiquement différentes. I. Matériel et méthodes. Bull. Inst. Roy. Sc. Nat. Bel., 47, 1-78.

VANDERSLEYEN, F'. (1967).- Atlas des Grottes de Belgique. Document de travail no5, Inst. Roy. Sc. Nat. Belg. 